\title{
Detection of Dyslexia using Eye Tracking Measures
}

\author{
Masooda Modak, Ketan Ghotane, Siddhanth V, Nachiket Kelkar, Aravind Iyer, Prachi G
}

\begin{abstract}
Dyslexia is one of the most common and hidden learning disabilities found in people, especially in the young age.It particularly affects reading, where the impaired reader takes a longer time to read and grasp the concept than the non-impaired reader.This further leads to academic failures. So studies to detect such issues have been conducted considering various factors like the reading times, fixation times, number of saccades(sudden movements in the eye), of both the impaired and non-impaired subjects, and give the best possible results.Thus, we plan to use the same eye tracking technique supported with machine learning models to detect and classify the individuals with and without dyslexia.The factors considered during the study are font-size, typeface, frequency of words(fixation times of non-impaired readers are more if frequency of encountered words is less) and age(people with learning disorders tend to enhance their reading skills with age), etc.
\end{abstract}

Index Terms-Dyslexia, eye tracking, eye movements, diagnosis, detection, prediction, machine learning, support vector machine.

\section{INTRODUCTION}

Dyslexia, which is a neuro developmental reading incapacity adversely affects the speed and accuracy of word recognition, and as an effect,delays reading fluency. Since reading ability is a talent that falls along a continuum,dyslexia is best considered as a barrier on this continuum with no clear-cut or absolute limit[9].Dyslexia happens in variable degrees of severity, and for that, ultimately, a subjective cutoff should be set on a nonstop variable so as to diagnose the disability[1].

Although the causes of learning disability don't seem to be absolutely understood,it primarily affects youngsters within the age class of 9-13.Early identification and knowledgeable skilled support is the only style of curation for kids with reading difficulties, and it's terribly troublesome to cure once recognizing youngsters formally diagnosed with a learning

Revised Version Manuscript Received on July 18, 2019.

Masooda Modak, Department of Computer Engineering SIES Graduate School Of Technology, Nerul, Navi, Mumbai, India. (Email: masooda.modak@siesgst.ac.in)

Ketan Ghotane, Department of Computer Engineering SIES Graduate School Of Technology, Nerul, Mumbai, India. (Email: ketan.ghotane15@siesgst.ac.in)

Siddhanth V, Department of Computer Engineering SIES Graduate School Of Technology, Nerul, Mumbai, India. (Email: siddhanth.v.1998@gmail.com)

Nachiket Kelkar, Department of Computer Engineering SIES Graduate School Of Technology, Nerul, Mumbai, India. (Email: nachiket.kelkar15@siesgst.ac.in)

Aravind Iyer, Department of Computer Engineering SIES Graduate School Of Technology, Nerul, Mumbai, India. (Email: siddhanth.v.1998@gmail.com)

Prachi G, Department of Computer Engineering Sardar Patel Institute of Technology, Andheri, Mumbai, India. (Email: masooda.modak@siesgst.ac.in) disability[9]. The average age for performing the diagnosis of a learning disability is thirteen years.By that age, it's not solely terribly troublesome for the students to cope-up to grade level in reading,the problems typically disturbs their overall performance which can cause psychological and emotional distress, lack of motivation and depression.

The LD detection centres are very few in cities and many times the parents find it infeasible to take the child to these centres for the LD detection test. Moreover, these tests are not of a day or two. It is a procedure involving at least two months for the detection.

There are some informal methods for detecting learning disability in which the child is supposed to fill in the forms or respond to some questionnaire. The problem with these approaches is that the child may himself be not aware that he is possessing such a difficulty or he may be reluctant or shy to accept the fact. Thus, there is a need for a system in which we avoid the child filling some forms or answering the questionnaires. The proposed work is an attempt towards this in which the child is given a task of reading and through the eye gaze the learning disability can be detected.

Quick and automated screening strategies based on key objective measurements of reading could help identifying people in danger of learning disability throughout the early school years.Current strategies, however, are restricted in measuring individual level of learning disability and is additionally not much efficient for detecting learning disability.Invariably, these tests need the subject to provide some specific response, generally under time pressure, like marking the word boundaries in sequences of words while not interword areas, providing words of various font sizes so as to detect their gaze time at the monitor.The outcome generates the proportion of correct responses which provides an estimation of the subject's performance in reading.By following eye movements throughout reading, we are able to offer accurate data regarding the subject i.e. whether or not diagnosed by learning disability.

More significantly,this way of measurement needs no alternative special response aside from the reading method itself and so makes it attainable to assess reading performance without any additional requirements from the subject. Also, this approach differs in several important ways from the screening strategies presently in use.Though it's been noted that the attention movements of dyslexic readers are completely different from those of traditional readers, other researches done also show that learning disability varies according to age[2]. 


\section{DETECTION OF DYSLEXIA USING EYE TRACKING MEASURES}

Using machine learning and predictive modeling,it is possible to detect from group-level descriptions to individual level predictions with high sensitivity and specificity,which is a first step towards making eye tracking a viable screening technique. Overall,eye tracking combined with machine learning may be used to develop quick,objective and accurate screening models helpful for identifying youngsters suffering from dyslexia.

\section{LITERATURE SURVEY}

The topic of using Machine Learning has always been a hot topic among the publishers of research papers.Among the wealth of resources available on the various technologies and methodologies available on making the various parts of our project functional,the following research papers were used as references.

Almost among all of the previous eye tracking experiments with people with and without dyslexia there have been differences found between populations suggesting that eye movements reflect the difficulties of individuals with dyslexia.In the paper,they have presented a statistical model to predict readers with and without dyslexia using eye tracking measures.Also,as Dyslexia varies according to age,the model,based on a Support Vector Machine binary classifier,detects and classifies among all the tested subjects using key informative features.Using

[3] we similarly used OGAMA Software to track the eye movements of the subject.

We are also using the Support Vector Machine and $\mathrm{K}$ Nearest Neighbours classifiers for comparison of accuracy. SVMs use a set of training examples to create a binary classifier, which involves selecting a function which will correctly classify given test examples as mentioned. A detailed implementation is given in [4].

\section{METHODOLOGY}

Traditional Video Oculography method for eye gaze detection is used in our experiment, According to [8], the following steps form a part of gaze point detection :

1. Image/Video Acquisition- Using an infrared webcam or an eye tracker connected to the computer.

2. Face Localization - Detecting a face in the image or the video and the extent of the face using template matching or haar like features.

3. Eye Localization - Detecting eyes in the face using template matching or corner detection methods.

4. Pupil Position Detection - Accurate positioning of the pupil, required for gaze point calculation, is performed using corner detection methods like the harris corner detector.

5. Eye Gaze Calculation - Eye metrics like fixations and saccades are calculated using velocity based or dispersion based algorithms.

Our experiment involves the following steps:-

\section{Input Gathering:-}

Normal text content would be provided for dyslexic as well as non-dyslexic people to read, with different text configurations like font-sizes, character spacings, and font-family for analyzing their behaviour.

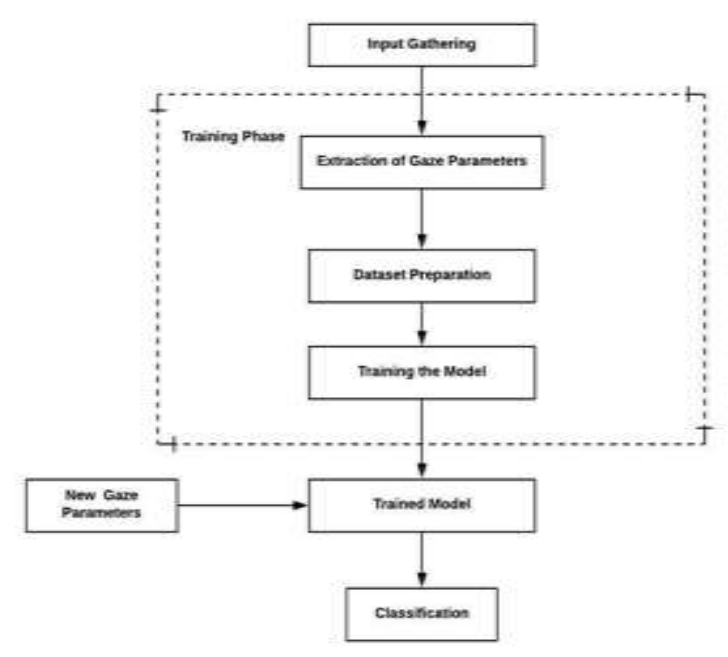

Fig. 3.1 Flowchart

\section{Extraction of Gaze Parameters:-}

Our system would use an image processing software called Open Gaze and Mouse Analyzer (OGAMA)[6]. The type of eye tracker used here is a screen based eye tracker, as explained in [7]. In this, the tracker would be on the screen or the display device. This software has the capabilities to make slideshows in which we would be presenting our content for the candidate to read, interface with an eye tracker or any standard webcam and calculate gaze parameters like fixations count, fixation time, saccade length and saccade velocity, etc. Fixations and saccades identification uses velocity and dispersion based algorithms mentioned in [5]. The modules under Open Gaze and Mouse Analyzer (OGAMA) that we used are[6]:-

\section{Recording Module:-}

The recording interface has been designed to capture subjects' gaze readings during the presentation of the experimental slideshow.

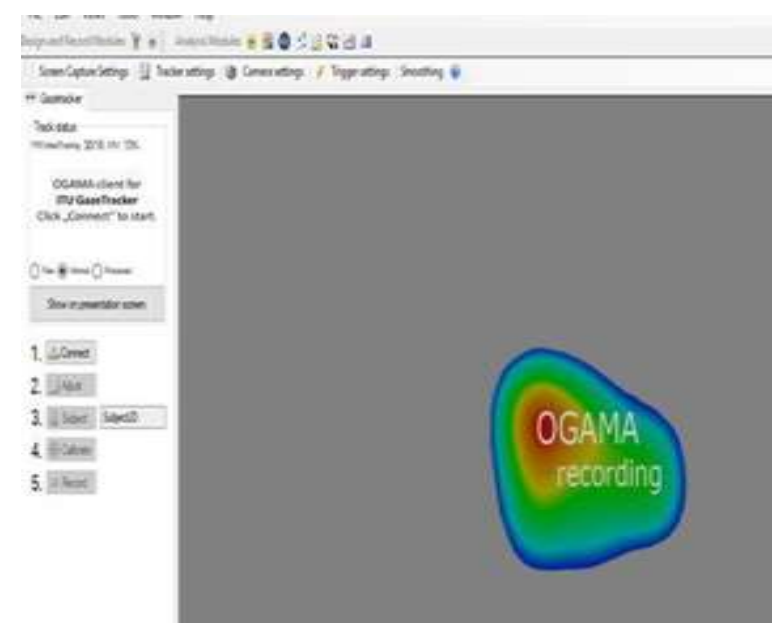

Fig. 3.2 Recording Module

\section{Replay Module:-}

The replay interface has been designed to replay the whole data logged during an experiment procedure. 


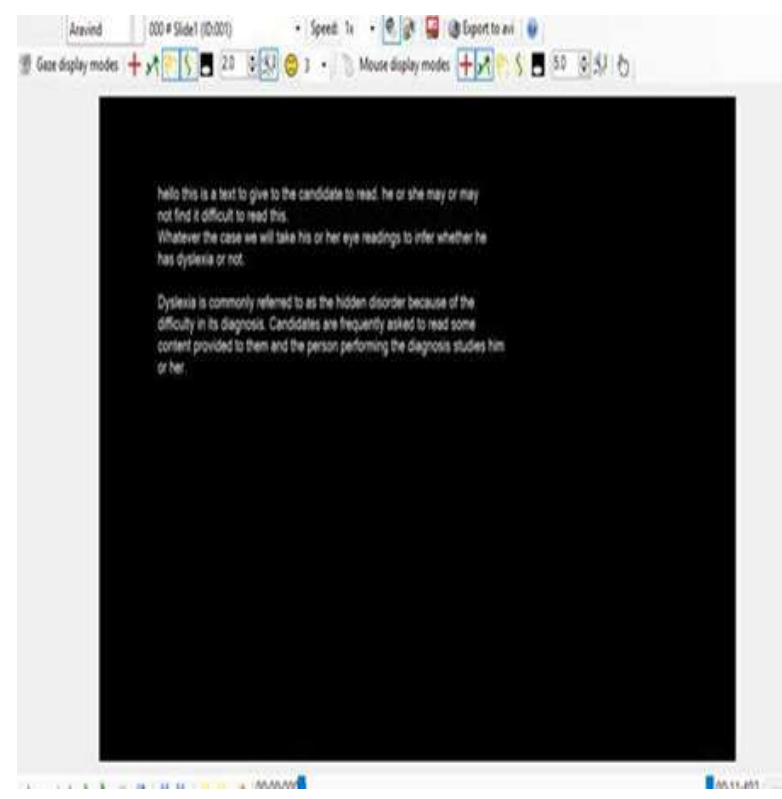

Fig. 3.3 Replay Module

Slideshow Module:-

The slideshow design interface allows the creation of slides to be presented during an experiment.

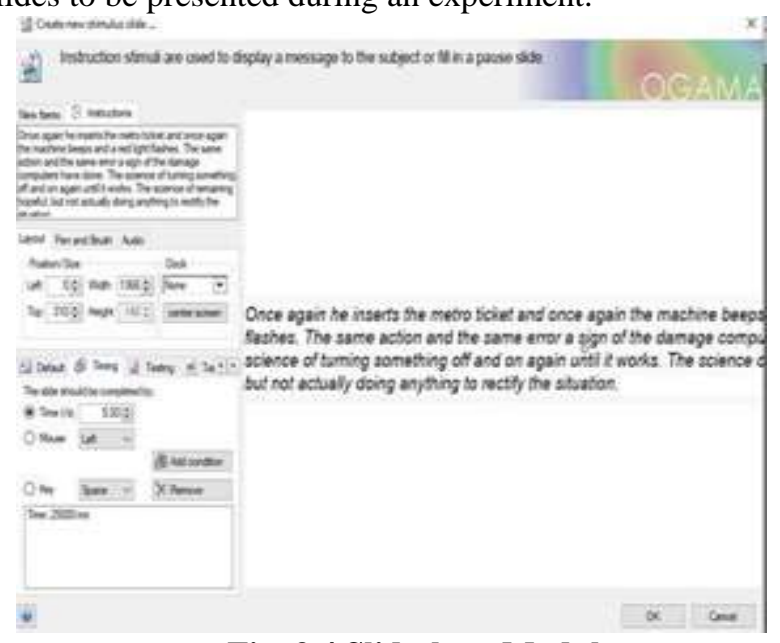

Fig. 3.4 Slideshow Module

Statistics Module:-

The statistics interface has been designed to calculate empirical parameters which are useful for further analysis.

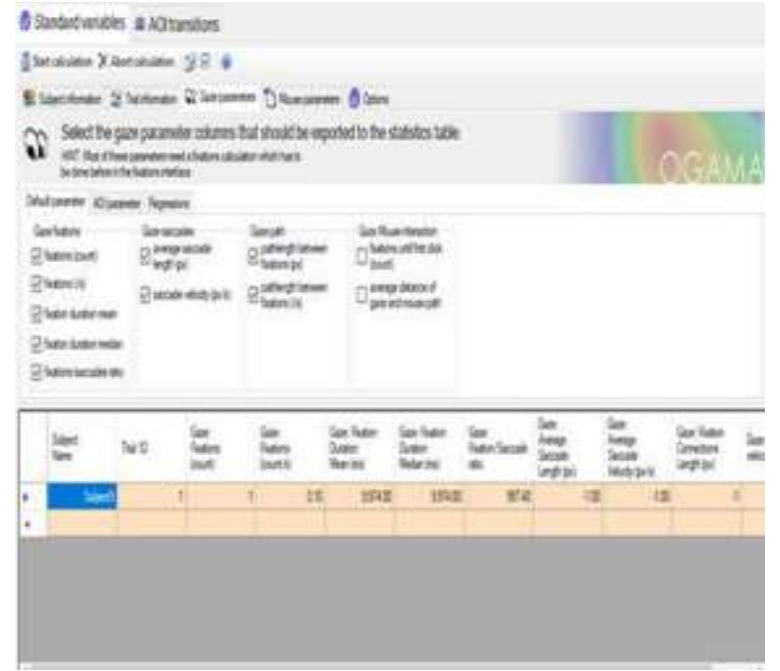

Fig. 3.5 Statistics Module

\section{Dataset Preparation and Training the Model:-}

The data obtained would be exported as a CSV file, which would further be used for training our model, along with the target value. The Support Vector Machine and K Nearest Neighbours classifiers would be used for training the model and the accuracy would be compared.

\section{Classification:-}

A new set of fixation and saccade readings would be generated, either from real time or by splitting the dataset into a training set and test set. This new set would be classified by the model.

\section{DATASET \& RESULTS}

Our dataset consists of a total of 33 readings out of which 15 belong to learning disabled candidates and the remaining 18 to normal candidates. Their ages ranged from 8 to 15 . We presented a set of 3 reading modules to each candidate. The 1 st two reading contents were paragraphs that were taken from school level reading passages and the 3 rd content was a poem. The font family used for the 1st two reading paragraphs was Arial with font sizes 34 and 23 respectively. For the 3rd content, we used Comic Sans MS as the font family with font size 24. A different foreground and background colour scheme was used for each reading content. A constant line spacing of 1.5 was used throughout.

The equipment used was a standard logitech webcam with a $1280 \times 720$ screen resolution.

Therefore, our dataset is composed of readings marked as LD if the participant has dyslexia and Non-LD if the participant does not have dyslexia.

The following features were extracted:-

1. Fixations( Count ) - Number of fixations recorded in the given time.

2. Fixations( Count / s ) - Number of fixations per unit time.

3. Fixation Duration Mean( ms ) - Sum of all fixation durations divided by fixation count.

4. Fixation Duration Median( ms ) - Median of all fixation times.

5. Fixation / Saccade Ratio - Sum of all fixation times divided by the total reading time.

6. Average Saccade Length ( px ) - Mean of all lengths of the straight line connections between consecutive fixations.

7. Average Saccade Velocity ( px / s ) - Mean of all saccade lengths /Timestamp Values.

Other commonly used parameters, given in [7] are:-

1. Scanpaths:-Sequences of Fixation - Saccade - Fixation movements.

2. Heat Maps:-Representation technique for displaying aggregations of gaze points and fixations.

3. Area of Interest:-Small Regions in the screen content defined by the user for extracting metrics specific to those regions.

These additional parameters were not used by our experiment.

For classification we used one random split of the data and 
K-fold cross validation, iterating 5 times. With the random split of the dataset into a training set of $90 \%$ and a test set of $10 \%$, got an accuracy of $75 \%$.we got an accuracy of $75 \%$.

With the cross validation procedure we got the following results:-

\begin{tabular}{|c|c|}
\hline Dataset & Accuracy \\
\hline Fold-1 & $71.43 \%$ \\
\hline Fold-2 & $57.14 \%$ \\
\hline Fold-3 & $57.14 \%$ \\
\hline Fold-4 & $42.86 \%$ \\
\hline Fold-5 & $80.00 \%$ \\
\hline
\end{tabular}

From the cross validation results for 5 iterations, we get an average accuracy of $61.74 \%$.

\begin{tabular}{|c|c|}
\hline Dataset & Accuracy \\
\hline Fold-1 & $80.00 \%$ \\
\hline Fold-2 & $40.00 \%$ \\
\hline Fold-3 & $80.00 \%$ \\
\hline Fold-4 & $40.00 \%$ \\
\hline Fold-5 & $80.00 \%$ \\
\hline Fold-6 & $40.00 \%$ \\
\hline Fold-7 & $100.00 \%$ \\
\hline
\end{tabular}

From the cross validation results for 7 iterations, we get an average accuracy of $65.714 \%$.

\section{CONCLUSION}

The eye movements of dyslexic people are different as compared to normal readers. People with dyslexia have a tendency of having longer fixations, they have longer reading times and make more fixations as compared to normal readers. In this paper we have shown how these characteristics can be integrated along with machine learning for the detection of dyslexia.

We have presented a method that takes the readings of candidates and predicts whether the candidate may have dyslexia or not. To the best of our knowledge, this is the first attempt to build a statistical model to predict automatically readers with dyslexia using eye tracking measures using OGAMA (Open Gaze and Mouse Analyzer) software. Our model calculates gaze parameters like fixations count, fixation time, saccade length and saccade velocity, etc.

Dyslexia is termed a hidden reading disability because it is laborious to diagnose. diagnosis dyslexia is crucial to deal with this condition however estimations of dyslexia are much more than the actual diagnosed population. Our model is simply a try on predicting dyslexia by employing a machine learning approach with eye tracking measures. However, we believe that it has an excellent impact.

Our study suggests that eye tracking measures have the potential to be used as an efficient method to diagnose dyslexia in the early stage itself before a sense of failure sets in into the student.Eye trackers are becoming more and more affordable and reading a text in silence is less intrusive than being exposed to the tests required in current diagnoses. Moreover, there are different human-computer interaction measures that were found to be associated with eye tracking measures, like mouse tracking, that are however yet to be explored. Their potential applications go from identification to user modelling via their interaction with the pc.

Eye tracking has a wide array of applications in other fields like Medicine, Neuroscience and Psychology, Gaming and Product Usability Testing. The eye metrics used in our experiment can be extended to be used in other comprehensive research work pertaining to eye tracking in the above mentioned fields[7].

The work can be extended to include the learning characteristics of the learner apart from eye gaze to detect the learning disability. Thus, the machine learning algorithm can then detect the learning disability depending on the overall learning characteristics along with eye gaze of the learner.

\section{REFERENCES}

1. Nilsson Benfatto M, qvist Seimyr G, Ygge J, Pansell T, Rydberg A, Jacobson C (2016) Screening for Dyslexia Using Eye Tracking during Reading. PLoS ONE 11(12): e0165508.

2. Rubino CA, Minden HA (1973) Analysis of eye-movements in children with reading disability. Cortex 9: 217220. Pmid:4744366

3. Detecting Readers with Dyslexia with eye tracking measures;Published by Luz Rello and Miguel Ballesteros in 2015 ACM.

4. Use of Support Vector Machines for Texture Classification;Published by Kwang In Kim, Keechul Jung, Se Hyun Park, and Hang Joon Kim in 2002 IEEE.

5. Identifying Fixations and Saccades in Eye-Tracking Protocols; Published by Dario D. Salvucci and Joseph H. Goldberg in 2000 ACM.

6. Vosskhler, Adrian Nordmeier, Volkhard Kuchinke, Lars \& Jacobs, Arthur. (2008). OGAMA (Open Gaze and Mouse Analyzer): Open-source software designed to analyze eye and mouse movements in slideshow study designs. Behavior research methods.40. 1150-62.10.3758/BRM.40.4.1150.

7. A Study of Eye Tracking Technology and its Applications; Published by Pramodini A. Pundem, Dr. Mukti E. Jadhav and Dr. Ramesh R. Manza in 2017 IEEE

8. Human Eye Tracking and Related Issues : A Review; by Hari Singh and Dr. Jaswinder Singh, in the International Journal of Science and Research Publications, 2012.

9. Adubasim ICJ, Nganji JT (2017) Dyslexia-A Learning Difference. Autism Open Access 7:203 doi:10.4172/2165-7890.1000203. 